ACTA UNIVERSITATIS LODZIENSIS

Folia Litteraria Romanica 14, 2019

http://dx.doi.org/10.18778/1505-9065.14.12

Małgorzata Posturzyńska-Bosko

Université Marie Curie-Skłodowska, Lublin

(iD https://orcid.org/0000-0002-1919-0065

m.posturzynska-bosko@poczta.umcs.lublin.pl

\title{
Analyse lexicale du vocabulaire concernant le fait de boire du vin d'après le Dictionnaire comique de Ph.-J. Le Roux (1786) et le Dictionnaire de l'Académie Française (1798)
}

\begin{abstract}
RESUMÉ
Le but de cet article est de montrer et d'analyser un répertoire des termes relatifs aux habitudes de boire du vin des Français à la fin du XVIII ${ }^{\mathrm{e}}$ siècle. L'analyse des données lexicales de deux dictionnaires de différentes conceptions lexicographiques, à savoir Dictionnaire comique, satyrique, critique, burlesque, libre et proverbial de Philibert-Joseph Le Roux de 1786 et Dictionnaire de l'Académie Française de

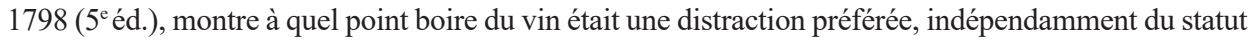
social. On trouve dans ces dictionnaires une surprenante richesse des termes décrivant la culture de la boisson, les types de vin, les rituels qui l'accompagnent, qui sont, dans la plupart des cas, des activités peu subtiles. Cette image précieuse est complétée par les expressions à l'aide desquelles on peut préciser le degré d'ivresse, les effets causés par le vin ; on y trouve aussi une panoplie de comportements après la consommation, une kyrielle de noms pour des vins de catégorie plutôt inférieure.
\end{abstract}

MOTS-CLÉS - culture de boire, dictionnaires du XVIII' ${ }^{e}$ siècle, vin

An Analysis of the Vocabulary Relating to Wine Drinking as Based on Dictionnaire comique by Philibert-Joseph Le Roux (1786) and Dictionnaire de l'Académie Française (1798)

\section{SUMMARY}

The purpose of this article is to present and analyse the terms found in two dictionaries published in the twilight of the $18^{\text {th }}$ century, namely Dictionnaire comique by Philibert-Joseph Le Roux (1786) and Dictionnaire de l'Académie Française (1798), both of which relate to the way that wine is drunk by the French. The plenitude of terms describing the drinking culture, the words for good and bad wine, the effects following wine consumption, the stages of alcohol intoxication, the names for persons consuming alcohol, and the rituals and drinking-related activities is accompanied by the socio-economic panorama. The two dictionaries have different lexicographical assumptions, i.e. while the Le Roux dictionary contains language material which often contradicts the principles of bel usage, the Academy's dictionary promotes the purity and correctness of the French language. Nonetheless, in most cases they give the same terms and expressions with regard to drinking.

KEYWORDS - drinking culture, dictionaries from the late $18^{\text {th }}$ century, wine 
Dans cet article, nous voulons présenter et analyser le vocabulaire contenu dans le Dictionnaire comique de Ph.-J. Le Roux et le Dictionnaire de l"Académie Française ( $5^{\mathrm{e}}$ éd.) concernant la culture de boire des Français à la fin du XVIII ${ }^{\mathrm{e}}$ siècle. Notre objet est avant tout de comparer la façon de présenter des mots et des expressions décrivant cette activité dans les dictionnaires de différentes conceptions lexicographiques. Dans le panorama lexicographique du XVIII siècle, le Dictionnaire comique de Le Roux occupe une place prééminente en tant que répertoire de la langue non conventionnelle qui répugne au bel usage. On y observe une insistance particulière sur les plaisirs de la vie, et sans doute, il est le plus riche en termes de gueule et de débauche assortis de commentaires piquants et amusants qui ont trait à l'action de boire, surtout du mauvais vin. Dans l'Avertissement l'auteur définit son public : son dictionnaire est pour tout le monde, il prône en quelque sorte, la démocratie de la lecture. Par contre, le but du Dictionnaire de l'Académie est de chercher à préserver en l'état la langue française, telle qu'elle devrait être écrite et parlée. Selon Y. Giraud (1983: 79, 85-86) :

On voit que le lexicographe se laisse souvent entraîner par une verve bavarde, voire lyrique et qu'il dépasse largement la sèche définition pour s'étendre sur la glose [...] Ainsi, on trouve réellement de tout dans ce Dictionnaire, à commencer par ce qu'on ne s'attend pas à rencontrer dans un ouvrage de cette sorte. Ce qui fait à la fois sa faiblesse et son intérêt. Car la lecture en est plaisante, et jamais fastidieuse ; on voit que Le Roux ne résiste pas au plaisir de développer certains commentaires satiriques de son cru. [...] C'est aussi un livre d'une lecture instructive, qui restitue un peu du parler de la conversation courante, qui est un répertoire fort précieux des habitudes de langage de deux ou trois générations. Il montre la richesse de la création verbale, aussi bien dans le monde populaire que dans la société raffinée, et il collectionne une foule de termes disparus depuis. C'est enfin un dictionnaire d'une lecture fort utile, « aux étrangers et aux Français mêmes », car il permet de comprendre ou de préciser le sens des tournures employées par les auteurs comiques, « réalistes » ou familiers [...].

Deux dictionnaires de conceptions lexicographiques différentes ne sont pas aussi ifférents que Le Roux le voulait. L'analyse du matériau montre qu'à peu près $80 \%$ du vocabulaire proposé par Le Roux est présent dans le Dictionnaire de l'Académie. Cependant, dans ce dernier, plusieurs explications des mots et des expressions diffèrent, elles sont atténuées pour la bienséance des lecteurs, le niveau de langue est précisé par les informations telles que : populaire, proverbialement, style familier, bas, injurieux, vulgaire, ce qui n'est pas présent dans le dictionnaire de Le Roux, sauf quelques rares exemples. Des centaines de mots et d'expressions (plus de 180 dans le dictionnaire de Le Roux sur plus de 1200 pages) et environ 140 occurrences trouvées dans le Dictionnaire de l'Académie sur 1544 pages) utilisés aussi bien dans le milieu populaire que dans la société raffinée, une collection enivrante et fastidieuse d'occurrences pour décrire les habitudes à table des bons vivants, qui jouissent de la vie en buvant surtout du vin de mauvaise qualité et dans des endroits qu'il ne faut pas fréquenter. 
Dans le Dictionnaire de l'Académie sous le terme vin, on trouve les informations de type encyclopédique, telles que le genre du vin (rouge, blanc), souches (muscat), leur origine (vin de Champagne), les récipients utilisés lors du processus de production et de consommation du vin (muid, quartaut), les activités décrivant les étapes de la production et de la conservation du vin (entonner). La plupart des occurrences décrivent la qualité, le bouquet, la couleur, le goût du vin, au total 89 pour décrire ces propriétés du vin, p. ex. : vin couleur d'œil de perdrix, sophistiqué, qui peche en couleur. En parlant de l'âge du vin, on disait : vin de deux feuilles, trois, quatre, etc. du vin qui a deux, trois, quatre ans respectivement. Le dictionnaire de Le Roux ne comporte pas ce type d'informations.

Nous avons classé le matériau recueilli en quelques groupes thématiques :

\section{Action de boire}

Boire est une action collective, inscrite dans un rituel social indépendemment de l'âge, de la position sociale, c'est une partie de l'identité des Français au XVIII siècle. Tout d'abord, boire est un plaisir, ce qui est plus ou moins explicitement exprimé dans les commentaires. Parmi les descriptions neutres de l'action de boire, on trouve : allumer la lampe 'verser du vin dans le verre de quelqu'un', brûler le fagot 'aller boire ensemble une bouteille au cabaret', amboire 'boire', lever le coude, mettre le pied dans la vigne du Seigneur (dans $\mathrm{A}^{1}$ pour 'être ivre'), faire la Saint-Martin, à cause des foires pour la vente du vin vers la Saint-Martin, le 11 novembre, ce qui signifiait 'boire à l'occasion des foires'.

\section{Plaisir de boire}

Le Roux présente quelques expressions dont l'explication met en valeur le plaisir de boire qui d'ailleurs rime très souvent avec excès : grenouiller 'ivrogner, être enfoncé dans un cabaret à buvailler comme grenouille dans l'eau'; l'auteur explique cette expression : 'barboter dans l'eau comme une grenouille'; de ce fait l'expression barboter dans le vin veut dire 's'ivrogner tout le long de la journée dans un cabaret' (A grenouiller 'ivrogner', pop.). Le verbe gobeloter est expliqué comme suit : 'grenouiller dans un cabaret' (sous le terme gobelotter, nous lisons dans A : 'boire à plusieurs petits coups'). Les trois expressions fioler, mesurer son vin et ramponer sont accompagnées de 's'enivrer à plaisir'. Le phraséologisme caresser la bouteille 'boire ou l'action de boire' a des connotations sensuelles, même sexuelles (il est absent dans A).

${ }^{1}$ Dans la suite de notre article nous utilisons l'abréviation A pour désigner le Dictionnaire de l'Académie, et l'abréviation LR - pour le dictionnaire de Le Roux. 


\section{Action excessive de boire}

Plus de 25 mots et expressions dans LR sont expliqués par : 'boire trop' : souffler la bougie, souffler la linotte, boire en lancement, à tire larigot, comme une éponge, comme un trou ('boire excessivement, à l'image d'un trou n'ayant pas de fond'). L'Académie précise ce dernier 'boire excessivement' prov.; comme synonymes, on a : à (en) tire larigot, comme un Templier, comme une éponge, s'enivrer, faire emplette de vin ('pour s'enivrer, boire plus que de raison', il est intéressant que dans A, cette expression signifie 'achat des marchandises', il n'y a aucune mention s'appliquant à l' action de boire du vin). Les expressions entrer en vin, flaconner, vuider les bouteilles et les flacons, hausser le gobelet, vuider les pots, fluter ('avaler du vin à pleins verres', ce que A explique en ces termes : pop. 'boire' sans préciser l'intensité), fouetter ('boire hardiment, beaucoup'), ce terme est accompagné des synomymes tels que lamper (A 'boire avidement', pop.), sabler (A 'avaler tout d'un trait', style fam.), entonner ('boire avec avidité sans apprécier le goût du vin', d'après $A$, d'un homme qui boit beaucoup), envermilloner, fouetter les poulets, poules, griser (A 'faire boire quelqu'un pour le rendre demi ivre'), s'humecter le pectoral, humetter, ausser le tems (A 'boire en débauche'), crapuler (A être dans la crapule 'vilain excès de boire et de manger', et l'expression : vivre dans la crapule 'se livrer sans choix et sans modération aux plaisirs de l'amour, de la table et du jeu'), pantagruéliser. Un verbe est défini dans LR comme vulgaire : chopiner (A 'boire du vin fréquemment', vulg.). Ce dernier verbe est à l'origine de l'expression : boire chopine sur chopine ('boire sans cesse'), mais A en donne la définition suivante : 'faire débauche de vin'. D'autres verbes, tels que : beuvailler ('boire avec excès, ivrogner, grenouiller'), beuvasser ('boire sans discontinuer comme font des ivrognes de profession') complètent la liste des expressions indiquant l'excès de boire. Deux expressions, d'après LR, viennent de la langue allemande : faire carousse 'avaler tout' de l'expression trinken gar aus 's'enivrer', ce que A explique : 'faire débauche, style fam., vieilli'. Le verbe suivant : trinquer, de l'all. Trinken, signifie 'boire avec excès'.

\section{Personnes buvant de l'alcool}

Ivrogne et ivre sont les plus fréquents pour nommer quelqu'un qui s'adonne à cette distraction (A définit ivrogne comme suit : 'qui est sujet à s'enivrer ou à boire avec excès'; cette distraction n'est pas étrangère au sexe féminin, à côté du terme masculin, on trouve ivrognesse 'femme sujette à s'ivrogner') ; les mots suivants complètent cette liste : biberon, biberonne (1. fam. A : 'celui qui aime le vin, et qui en boit volontiers'). Des termes mentionnés : imprimé, pochillateur, fesse pinte, gavache, gotzi, imbriaque (de cette série, ce dernier imbriaque est dans A 'mot pop. pour un homme, qui pour avoir trop bu, a perdu la raison'), rébus, 
vineux (dans A, cet adjectif s'applique plutôt à la force du vin, le goût, l'odeur, la couleur, et non à une personne s'adonnant à boire), vadele, averlant : tous ces mots sont accompagnés dans LR du mot ivre, les termes suivants ont des explications supplémentaires : piffre 'ivre, qui n'est jamais sou, goulu' (d'après A, ce terme est bas et injurieux pour des personnes excessivement grosses). Les termes : rissole 'sobriquet qu'on donne à un ivrogne' (A 'sorte de menue patisserie'), frere de jubilation veut dire 'un homme gai, un ivrogne, un buveur, un homme sans souci', d'après A : 'réjouissance, bonne chère', ne signifient pas être ivre. La comparaison des expressions nous donne les conclusions suivantes : celui qui a bien bu - 'en a autant qu'il lui en faut, être dans les broussailles', c'est la même chose que : être complet, dessous, être imbu du vin, être sac à vin, être dans les vignes. Le Roux explique aussi qu'une personne ivre ou boite, généralement, est en état moins grave qu'une personne enivrée ou chopine parce que les premiers signifient quelqu'un qui a la raison brouillée, les suivants signifient que le vin lui fait perdre la raison. Ivre comme une soupe, ivre mort signifient 'être extrêmement ivre'. Dans ce dictionnaire, à côté des expressions définissant l'état d'une personne qui boit, on trouve encore celles qui renvoient à la mythologie et au culte ancien de Bacchus : être Bacchus, disciple ou enfant de Bacchus, Dieu de vin ou suppôt de Bacchus. Elles définissent l'amateur des boissons, et barbe fleurie, de Bacchus, c'est 'un buveur à rouge trogne qui à force de boire a la face fleurie et enluminée'. Deux syntagmes nominaux désignent les amateurs de boissons à valeur neutre, sans les nommer ivrognes : chevalier de la coupe 'celui qui aime boire pendant des débauches honnêtes' (but : pour oublier les ennuis) ; l'expression être en pointe de vin signifie quelqu'un qui a 'un peu de vin dans la tête', est 'un peu échauffé par le vin'. Sous le terme vin dans A, on trouvera aussi la gradation, en quelque sorte, de l'état des amateurs de boissons après la consommation : un homme est en pointe de vin pour que le vin commence à le mettre en gaité (LR), il est chaud de vin pour dire qu'il commence à être ivre, il est pris de vin qu'il est déjà ivre et il est entre deux vins qu'il approche de l'ivresse. D'un grand ivrogne on dit qu'il est sac à vin (LR), et d'un homme qui est extrêmement ivre : le vin lui sort par les yeux.

\section{Vin - caractéristique}

Avant tout le vin est une boisson préférée, chérie ; quant aux autres boissons alcoolisées, il n'y a que peu d'occurrences autres que le vin : biere qui apparaît dans un jeu de mots : Les ivrognes disent qu'ils ne veulent point mettre leur corps en biere pour dire : boire de la biere au lieu de vin (A 'espèce de boisson forte, qui se fait de blé, d'orge ou de houblon'), et coco 'eau de vie à la base du vin', ce que A précise : 'liqueur bonne à boire'. En France, la culture de boire du vin s'inscrivait dans la tradition chrétienne, elle en était un élément indissociable, alors accepté jusqu'à tel point que quelqu'un qui buvait de l'eau 
était considéré comme homme froid (buveur d'eau 'homme froid'). D'ailleurs, ivrogne, il ne hait rien tant que l'eau. Celui qui mange sans boire, mange le repas de brebis. On attribue au vin des propriétés bienfaisantes : un verre de vin avise bien un homme, un ivrogne dit du vin, après Dieu, voilà mon sauveur ; Le Roux cite d'après le roi d'Arragon, Alphonse le Sage : il n'y a rien de meilleur que d'avoir du vieux bois pour brûler, du vin vieux pour boire, des vieux amis pour faire société et de vieux livres pour lire et le reste n'est que babioles, de plus, le vin donne de la force: du vin n'a que l'épée et la cape 'qui a peu de vin, peu de force' A.

Le vin en termes génériques, c'est : eau bénite de cave, souveraine gomme, jus de Bacchus, jus de la grappe, jus d'octobre, jus de la vigne (A), jus du bois tortu, liqueur bachique, pianche, piot (A terme dont on se sert en plaisanterie), lait des vieillards. Pour exprimer la bonne qualité du vin, on dit : vin qui rappelle à boire, vin à fendre des pierres, vin d'une oreille mais vin de deux oreilles était au contraire un mauvais vin (A vin d'une oreille et de deux oreilles sont définis comme fam., sans préciser la qualité du vin).

Le nombre d'occurrences pour nommer le mauvais vin n'est pas symétrique par rapport aux locutions pour le bon vin. Cette inégalité, peut être, confirme que la fréquence de boire de ce premier était beaucoup plus élévée : on buvait mauvais vin, méchant vin (A 'qui ne vaut rien dans son genre'), ripopé ('mélange que font les Cabaretiers de différens restes de vin'), guinguet, chasse-cousin (A 'méchant vin'), casse-tête, racle-boyau (A. d'un vin trop vert), vin de Bretigny (selon LR, le pire en France : on disait c'est du vin de Bretigny qui fait danser les chèvres), vin de gargote à quatre sols la bouteille (A gargoter 'hanter les méchants petits cabarets'), pissotiere (A 'une fontaine qui jette peu d'eau'). On disait aussi pour s'excuser de la consommation du mauvais vin : le vin trouble ne casse point les dents. La mauvaise qualité du vin très souvent est due aux actions malhonnêtes des taverniers qui avaient l'habitude de : baptiser le vin 'mettre de l'eau dans le vin'. On a même institué des contrôleurs - rats de cave - pour vérifier si le vin n'était pas aqueux (A ‘commis des aides qui visitent le vin dans les caves').

\section{Effets après la consommation}

Une série d'expressions 'animalières' du vin définit les effets consécutifs à la consommation : après avoir bu du vin de singe on est gai et de bonne humeur, vin d'âne assoupit les sens, vin de cerf rend mélancolique et provoque les larmes, vin de lion rend querelleur, vin de pie rend agité, bavard, vin de porc fait vomir, vin de renard rend fourbe et fin. Une expression décrit aussi un effet particulier, quand le vin sort par les narines, c'est du vin de Nazareth. Pour les effets causés par le vin, A précise : 'on dit figurément d'un homme 
qui devient querelleur quand il a bu : il a le vin mauvais, qui est triste ou gai, c'est celui qui : a le vin triste ou gai respectivement. La liste des effets après la consommation excessive est longue : étourdissement, maladie vineuse, mal ou maladie $S$. Martin, celui qui a trop bu fait des esses, il va en serpentant à la maniere d'un $S$. (A 'on chancelle'), il a un soupir d'ivrogne, une haleine vineuse, il arrive qu'il vomisse : écorche le Renard (A bas), rend gorge (A), dégobille (A bas), joue à la corbette; pour guérir la gueule de bois, il doit prendre du poil de la bête 'boire un verre' (A 'il faut chercher son remède dans la chose même qui a causé le mal'). L'excès du vin affecte la beauté, parce que la personne qui boit a le nez : d'un ivrogne, de pompettes, à boire au baril, fleuri, enluminé, de betterave (ce dernier est aussi présent dans A).

\section{Rituels et habitudes}

Avant de boire, on carillonne, on choque les verres, on tinte (A 'faire tinter un verre, lui faire rendre un son en le frappant'), on toque (A), en buvant, on criait vive la joie, à brum, huzza. Celui qui voulait remercier, faisait raison (A 'lorsqu'un homme boit une santé qu'on lui a portée'). Le Roux décrit un rituel appelé faire rubis sur l'ongle, qui 'se pratiquait en débauche' : après avoir 'bu une rasade à la santé d'une personne de la compagnie, ou d'une autre qui est absente ou qu'on aime ou estime, on renverse la dernière goutte qui demeure dans le verre sur l'ongle du pouce, et ensuite on lèche cette même goutte, pour marquer l'attachement qu'on a pour la personne' (A mentionne ce rituel, sans en expliquer les raisons). Le dernier verre bu à la fin de la rencontre, c'était coup de l'étrier (A 'le vin que l'on donne au départ, lorsque quelqu'un est prêt à monter à cheval'). Les deux dictionnaires contiennent l'expression : cuver son vin dont l'acception est 'dormir afin de laisser passer son ivresse'.

\section{Conclusion}

Le dictionnaire de Le Roux montre la richesse de la création verbale, aussi bien dans le monde populaire que dans la société raffinée, et il collectionne une foule de termes qu'on peut trouver également dans le dictionnaire de l'Académie, classés vieillis, familiers, vulgaires, proverbiaux. Si le second explique aux lecteurs les règles de ce fameux concept du bon usage, le premier lui donne la joie de découvrir des mots et des tournures si vivants et gais, mais en même temps authentiques sans les proscrire ni les déterminer négativement, et son intérêt est de permettre au lecteur de savourer cette richesse, de se plonger dans ce monde linguistique sans remords. Il est opportun de constater que le dictonnaire de l'Académie, s'il n'estime pas cette création, il la fait voir. 


\section{Bibliographie}

Dictionnaire de l'Académie françoise, $5^{\mathrm{e} e ́ d ., ~ P a r i s, ~ 1798, ~ h t t p: / / a r t f l . a t i l f . f r / d i c t i o n n a i r e s / a c a d e m i e / ~}$ cinquieme/cinquieme.fr.html, consulté : 29.05.2008

Giraud, Yves, "Le Dictionnaire comique de Le Roux (1718) ", Cahiers de l'Association internationale des études francaises, $1983, \mathrm{n}^{\circ} 35, \mathrm{p} .69-86$

Le Roux, Joseph-Philibert, Dictionnaire comique, satyrique, critique, burlesque, libre et proverbial, vol. 1-2, Pampelune (Paris), 1786

Małgorzata Posturzyńska-Bosko - docteure habilitée, professeure adjointe à l'Institut de la Philologie Romane de 1'Université Marie Curie-Skłodowska à Lublin. Thèse de doctorat : Les Anaphores associatives dans les textes politiques de Christine de Pizan (2004), thèse d'habilitation : La Dynamique du système des pronoms personnels en moyen français : exemple des textes de Christine de Pizan (2017). Domaines de recherche : linguistique diachronique, ancien et moyen français, littérature médiévale, anciens dictionnaires. 